\title{
Tracción quirúrgica de canino retenido maxilar asociado a la presencia de un odontoma, diagnóstico radiográfico. Reporte de un caso
}

\author{
Surgical traction of retained maxillary canine associated with the presence of an \\ odontoma, radiographic diagnosis. Case report
}

María Ullaguari-Landeta (D) $^{\mathrm{a}}$, Alex Cruz-Gallegos ${ }^{\mathrm{a}}$

\begin{abstract}
RESUMEN
El odontoma es un tumor benigno mixto de origen odontogénico aunque por su comportamiento corresponde a un hamartoma, el cual está compuesto por células mesenquimatosas y epiteliales diferenciadas, haciéndolo de un aspecto normal pero de estructura defectuosa. Este tipo de lesiones se descubren con el examen radiográfico de rutina, el motivo de consulta más habitual es la falta de erupción de un diente permanente, con o sin la persistencia del diente temporal. En este artículo se presenta un caso quirúrgico-ortodóncico de un canino superior impactado en un paciente pediátrico, acompañado de la extracción de un odontoma, con un completo diagnóstico clínico y radiográfico (tomografía). Se presenta el caso de una paciente pediátrica de 12 años de edad con retención dentaria del canino superior derecho, causada por la presencia de un odontoma compuesto. Se realizan diversos estudios radiográficos para su diagnóstico, que consiste en radiografía panorámica y radiografías intraorales. Se realiza tratamiento conjunto ortodóntico quirúrgico.
\end{abstract}

Palabras clave: Odontoma; Diente canino; Anomalías dentarias. (Fuente: DeCS BIREME)

\section{ABSTRACT}

The odontoma is a mixed benign tumor of odontogenic origin although its behavior corresponds to a hamartoma, which is composed of differentiated mesenchymal and epithelial cells, making it a normal appearance but with a defective structure. This type of lesions are discovered with the routine radiographic examination, the most common reason for consultation is the lack of eruption of a permanent tooth, with or without the persistence of the temporal tooth. In this article we present a surgical-orthodontic case of an impacted upper canine in a pediatric patient, accompanied by the extraction of an odontoma, with a complete clinical and radiographic diagnosis (tomography). We present the case of a pediatric patient of 12 years of age with dental retention of the right upper canine, caused by the presence of a compound odontoma. Various radiographic studies are carried out for its diagnosis, consisting of panoramic radiography and intraoral radiographs. Surgical orthodontic joint treatment is performed.

Key words: Odontoma; Cuspid; Tooth abnormalities. (Source: MeSH NLM) licencia Creative Commons Atribución 4.0 Internacional (CC BY 4.0)

https://creativecommons.org/licenses/by/4.0/deed.es

Correspondencia:

Alex Cruz

Correo electrónico: alexandercruz1993@hotmail.com

Dirección: Quito-Ecuador

Citar como: Ullaguari Landeta M, Cruz A. Tracción quirúrgica de canino retenido maxilar asociado a la presencia de un odontoma, diagnóstico radiográfico. Reporte de un caso. KIRU. 2021;18(1):32-39. https://doi.org/10.24265/kiru.2021.v18n1.05 


\section{INTRODUCCIÓN}

El término "odontoma" fue utilizado por primera vez por Paul Broca en 1867 originariamente usado para nombrar cualquier tipo de neoplasia de origen odontogénico" (1). Actualmente el término odontoma a pesar que por su comportamiento corresponde a un hamartoma, se define como tumor benigno mixto de origen odontogénico, el cual está compuesto por células mesenquimatosas y epiteliales diferenciadas, haciéndolo de una apariencia normal, pero de estructura defectuosa ${ }^{(2)}$.

Varios autores han propuesto diversas clasificaciones sobre los odontomas, Sutton ${ }^{(3)}$ los clasifica en: odontomas quísticos y sólidos ; Bashkar (3) los clasifica en compuestos, complejos y quísticos; sin embargo la clasificación aceptada actualmente es la propuesta por la Organización mundial de la Salud $^{(4)}$ quien clasifica al odontoma dentro de los tumores benignos de epitelio odontogénico con ectomesénquima odontogénico con o sin formación de tejido dentario duro en: odontomas compuestos y odontomas complejos, ésta clasificación la realiza teniendo en cuenta la organización y el grado de alteración en la morfodiferenciación de las células odontogénicas.

Según Harris (2) el odontoma compuesto está formado por múltiples estructuras semejantes a dientes pequeños, conocidos como dentículos, dispuestos en un patrón ordenado, radiográficamente se observa una imagen mixta radiolúcida y radiopaca con bordes radiopacos definidos, múltiples zonas radiopacas en su interior de diversos tamaños. Mientras que en el odontoma complejo se presentan conglomeraciones amorfas de tejido odontogénico dispuestos en un patrón desordenado dificultando su diferenciación, radiográficamente se observa como una masa amorfa radiopaca, difusa con un halo radiolúcido.

En cuanto a la etiología de los odontomas hay diferentes factores que se mencionan en la literatura, "se les asocia con antecedentes previos de traumatismos durante la primera dentición, así como a procesos inflamatorios o infecciosos, anomalías hereditarias (síndrome de Gardner, síndrome de Hermann), hiperactividad odontoblástica o alteraciones en el gen de control de desarrollo" (5), mientras que otros autores ${ }^{(2,3,6)}$ adicionalmente relacionan a los restos paradentales de Malassez como parte de la etiología de los odontomas.
Respecto a su localización "hay una predilección en el maxilar del $67,4 \%$ y en la mandíbula del $32.6 \%$ " (1); mientras que "hay una predilección de $57,5 \%$ en maxilar y $42,5 \%$ en mandíbula" (7). Para otros autores "los odontomas compuestos principalmente se localizan en la región anterior del maxilar superior y los odontomas complejos principalmente se localizan en la región posterior de la mandíbula" ${ }^{(2,8)}$.

Por lo general el diagnóstico de éstas lesiones es casual, en su mayoría, acompañadas de la falta de erupción dentaria, "estas lesiones se descubren con el examen radiográfico de rutina, el motivo de consulta más habitual es la falta de erupción de un diente permanente, con o sin la persistencia del diente temporal" (2). Palacios (1) afirma que se diagnostican de forma casual mediante exámenes radiográficos de rutina entre la segunda y tercera década de la vida o bien, a partir de algún signo clínico como retraso de la erupción dentaria, afín con los anteriores autores,

Flores et al (9) menciona que los odontomas suelen ser asintomáticos y su hallazgo suele ser casual tras realizar un estudio radiográfico habitual, generalmente entre la primera y la segunda década de la vida; aunque "pueden aparecer signos y síntomas relacionados con su presencia como son dientes supernumerarios, incisivos impactados, caninos impactados, molares impactados, inflamación e infección, obstrucción nasal" ${ }^{(5)}$. A pesar de ser un hallazgo hay que realizar una adecuada anamnesis $y$ correlacionar signos que puedan llevar a un diagnóstico correcto. Tal como lo menciona Moya, (6) el diagnóstico se obtiene por la correlación de antecedentes relevantes, el examen clínico y radiográfico, siendo necesario realizar un diagnóstico diferencial para diferenciar los odontomas compuestos de los complejos y confirmar el diagnóstico definitivo con el análisis histopatológico.

Como se menciona anteriormente uno de los signos relacionados con la presencia de odontoma, es la presencia de caninos impactados, en el presente trabajo se lo denominará como canino incluido ya que éste término según Escoda ${ }^{(10)}$ hace referencia a aquellos caninos que no erupcionan, y permanecen dentro del maxilar más allá de su edad de erupción normal, a pesar de tener la raíz completamente formada. Sobre la frecuencia de dientes incluidos, según la mayoría de autores, el canino superior ocupa el segundo lugar, solo tras del tercer molar inferior. Según BertenCieszynski (citado por Ries Centeno), la 
frecuencia de caninos superiores incluidos es de $34 \%$ y de caninos inferiores $4 \%$. Mientras en lo que se refiere a la distribución por sexo, existe una clara predilección por las mujeres, con una frecuencia de 1,5 a 3,5 veces mayor que en los hombres ${ }^{(10)}$.

En cuanto a la etiología del canino retenido se debe a varios factores tales como: "Involución de maxilares, trayecto de erupción largo y complejo, con orientación a menudo desfavorable, patología tumoral como el odontoma, traumatismos, alteraciones de los incisivos laterales u origen genético" (10).

Para el diagnóstico es de suma importancia realizar un correcto estudio clínico-radiológico, aunque "el diagnóstico de inclusión se suele hacer de forma tardía" (10). Debe tenerse en cuenta que hay signos indirectos que pueden conducirnos a la sospecha de la presencia de inclusión de caninos como son: "presencia del canino temporal en la arcada dentaria, persistencia del espacio que debería ocupar el canino permanente, desplazamiento de los dientes vecinos, relieves de la mucosa, fistulas, cambios en la coloración de los dientes vecinos" (10)

Después de haber realizado la inspección clínica se debe confirmar la presencia de un canino retenido, a través de un estudio radiográfico. Se dispone de una serie de técnicas como son: radiografía panorámica, radiografía periapical, radiografía oclusal, radiografía lateral de cráneo y tomografía.

Según Escoda ${ }^{(10)}$, a través de un adecuado estudio radiográfico, se puede observar lo siguiente:

- La posición respecto de la arcada dentaria en los tres planos del espacio.

- Relación con los dientes vecinos (incisivos y premolares) y con las estructuras anatómicas vecinas importantes (seno maxilar, agujero palatino anterior, fosas nasales, agujero mentoniano).

- Orientación y situación de la corona y el ápice; complicaciones con significación radiológica (infecciosas, tumorales, mecánicas).

- Estado de las estructuras periodontales (hueso alveolar, espacio del ligamento periodontal, presencia o no de anquilosis).
- Forma, tamaño y localización del diente y tipo de tejido óseo que rodea el canino incluido.

Una vez concluido tanto el estudio clínico como el radiográfico, se establece el diagnóstico y se planifica el tratamiento ${ }^{(10)}$.

El creciente conocimiento de la importancia del canino, tanto en el aspecto estético, como de su función y en la oclusión, produjo una modificación progresiva de los conceptos terapéuticos y se adoptó una actitud más conservadora, orientada a conducir el diente a la arcada e integrarlo a las funciones propias de su posición y condiciones anatómicas.

Los autores Harris y Blanco ${ }^{(2,3,5)}$ sugieren un tratamiento netamente quirúrgico, incluyendo enucleación, eliminando la cápsula de tejido conjuntivo que lo rodea al odontoma. Mientras que otros autores como Flores et al ${ }^{(9)}$; Thistle, et al (11) sugieren un tratamiento quirúrgicoortodóncico: quirúrgico para el odontoma y ortodóncico para el canino incluido el cual se reposicionará en la arcada.

El objetivo de este trabajo fue exponer el manejo y evolución de un caso de odontoma en paciente pediátrico asociado a canino incluido maxilar, en el cual se realizó un diagnóstico radiográfico y un tratamiento quirúrgico.

\section{CASO CLÍNICO}

Paciente sexo femenino de 12 años de edad, sistémicamente sana. La cual es llevada a las clínicas odontológicas de pregrado de la Universidad Tecnológica Equinoccial en QuitoEcuador. El motivo de consulta fue "a mi hija aun no le sale el canino y quiero que le revisen". Al ser una menor de edad, se le informo a la madre sobre el tratamiento a realizar y se le solicitó firmar el consentimiento informado en el cual autorizaba el análisis clínico y radiográfico, el tratamiento clínico, farmacológico y quirúrgico, así como su difusión en relato de caso clínico. Se cumplieron los principios éticos y de confidencialidad exigidos en estos casos.

Se clasificó a la paciente como braquifacial, clase I esqueletal. En el examen intraoral, se encontró a la palpación, un aumento de volumen en la zona vestibular superior a la línea mucogingival, por vestibular de la pieza1.2, sin embargo la paciente no relataba dolor o sensibilidad en esta zona. Figura 1. Al examen radiográfico se identificó la presencia del canino maxilar derecho retenido (1.3), el cual presentaba una posición 


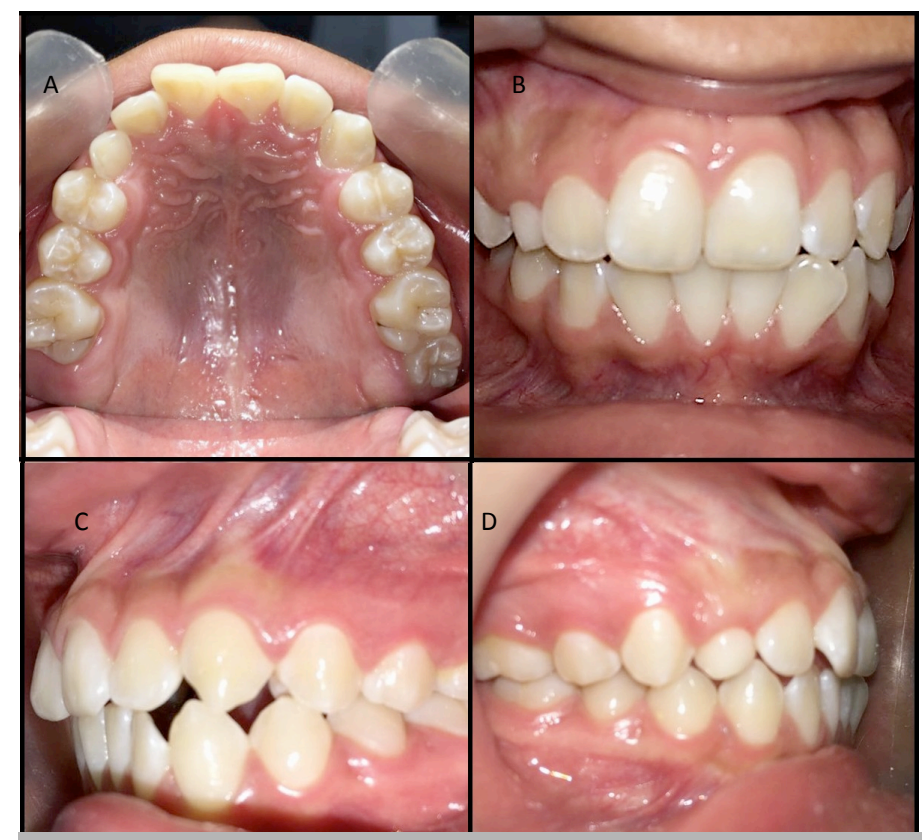

Figura 1. Fotografias intraorales $A, B, C, D$

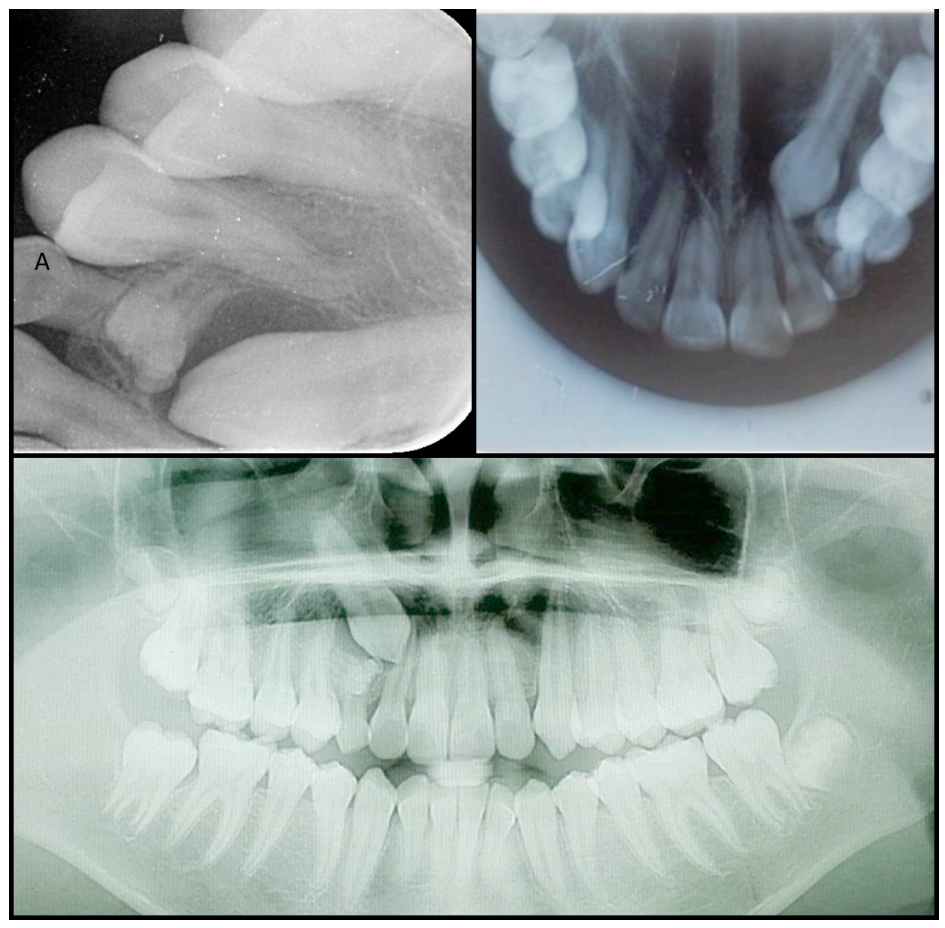

Figura 2. (A)Radiografía periapical, se muestra el nivel de inclusión del canino (1.3). (B) Radiografía oclusal, se observa el canino en dirección oblícua hacia los ápices las piezas 1.1 y 1.2. (C) Radiografía panorámica, nótese la presencia del canino deciduo en posición del canino definitivo, y la presencia del odontoma. 


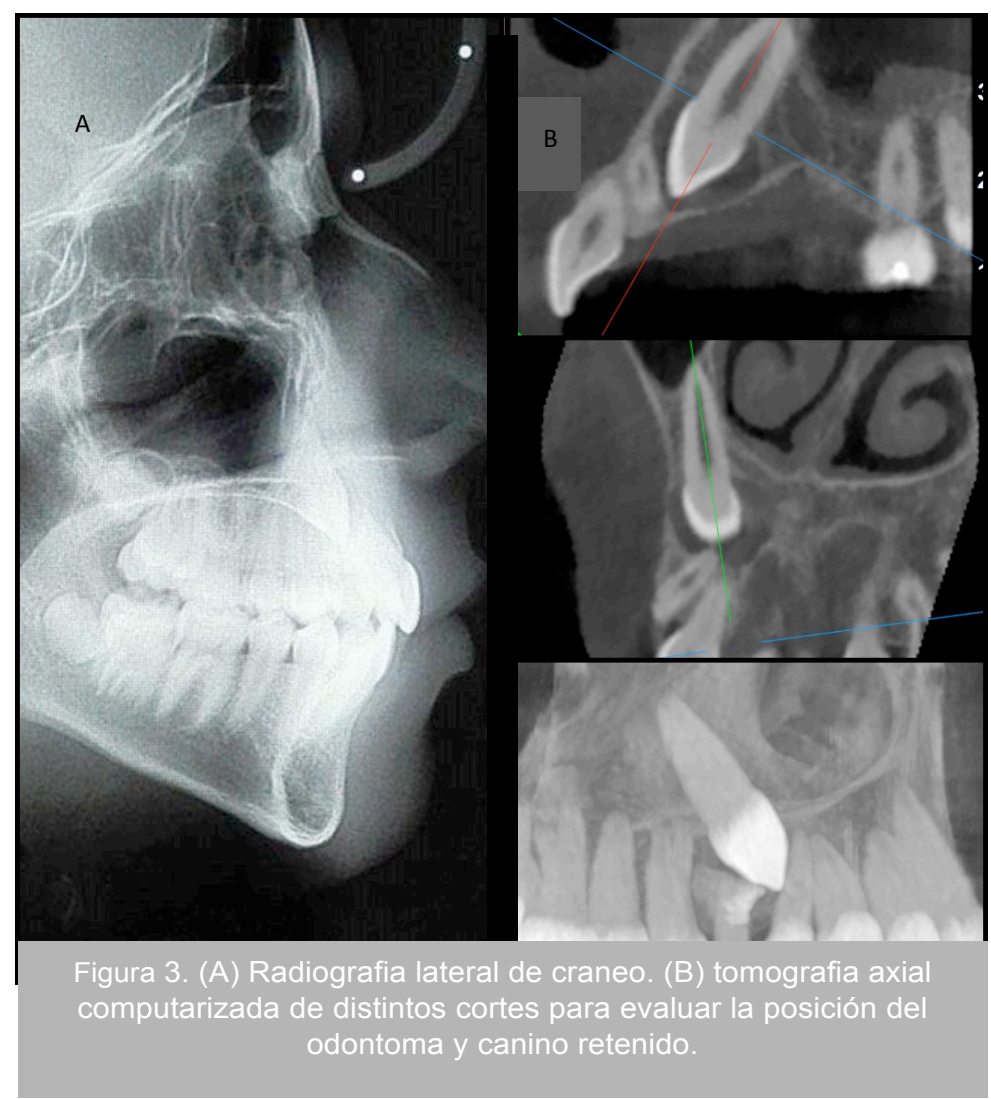

oblicua en relación a los ápices del incisivo central y lateral maxilares, derechos (1.1 y 1.2), un odontoma y canino temporal (pieza 53). Se realizó también una radiografía lateral de cráneo $y$ un estudio tomográfíco para un mejor diagnóstico y localización de la pieza retenida. Figura 3. El diagnóstico fue odontoma y canino maxilar retenido (pieza 1.3).

El plan de tratamiento se inició con la primera fase, que consistía en la eliminación de placa dentobacteriana e instrucción de técnica de cepillado. También restauraciones en resina fotocurable de las piezas 1.6 y 2.6 . Sellantes en las piezas $1.5,2.5,3.4,3.5,3,6,4.5,4.6$.

En la segunda fase, se realizaron las exodoncias indicadas por el ortodoncista, de las piezas $2.4,3.4,4.4$, acompañado de la colocación de ortodoncia fija MBT 022.

En la tercera fase se extrajo el canino temporal. Posteriormente se realizo un colgajo de espesor total, con el diseño de dos descargas, con preservación de papilas para los dientes vecinos. Se decidió éste abordaje por el nivel de inclusión del diente y por la facilidad de exposición que podemos lograr con este acceso.

Se extrajo el odontoma sin complicaciones. Una vez localizado el sitio anatómico en relación a la corona del canino retenido, se decidió realizar una osteotomía controlada con abundante irrigación a través de suero salino. Se utilizó una fresa redonda diamantada de baja velocidad hasta exponer el tejido pericoronario, que fue removido con periostótomo biselado. La exposición se extendió hasta $9 \mathrm{~mm}$. y se procedió a la cementación de un bracket para tracción en la porción coronaria expuesta del canino. Una vez realizado el montaje ortodóntico se trabajo el arco superior con arco de nitinol clásico 0.14 y se utilizó un kit adhesivo y resina transbond $\mathrm{XT}$, $3 \mathrm{M} \circledast$. Para realizar la tracción del canino se unió un alambre de acero 0.8 que quedó dentro del colgajo levantado por el cirujano. Figuras 4 y 5 . 


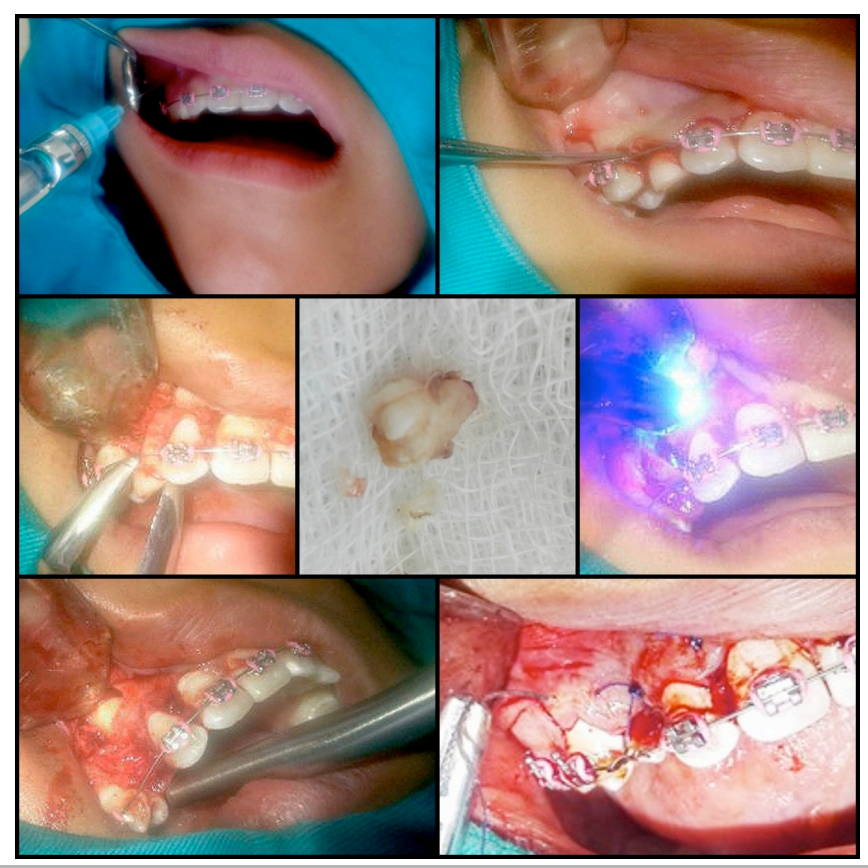

Figura 4. Localización de la porción coronaria del canino maxilar permanente

(1.3), luego de realizar una osteotomía controlada de $9 \mathrm{~mm}$., para la cementación del bracket.

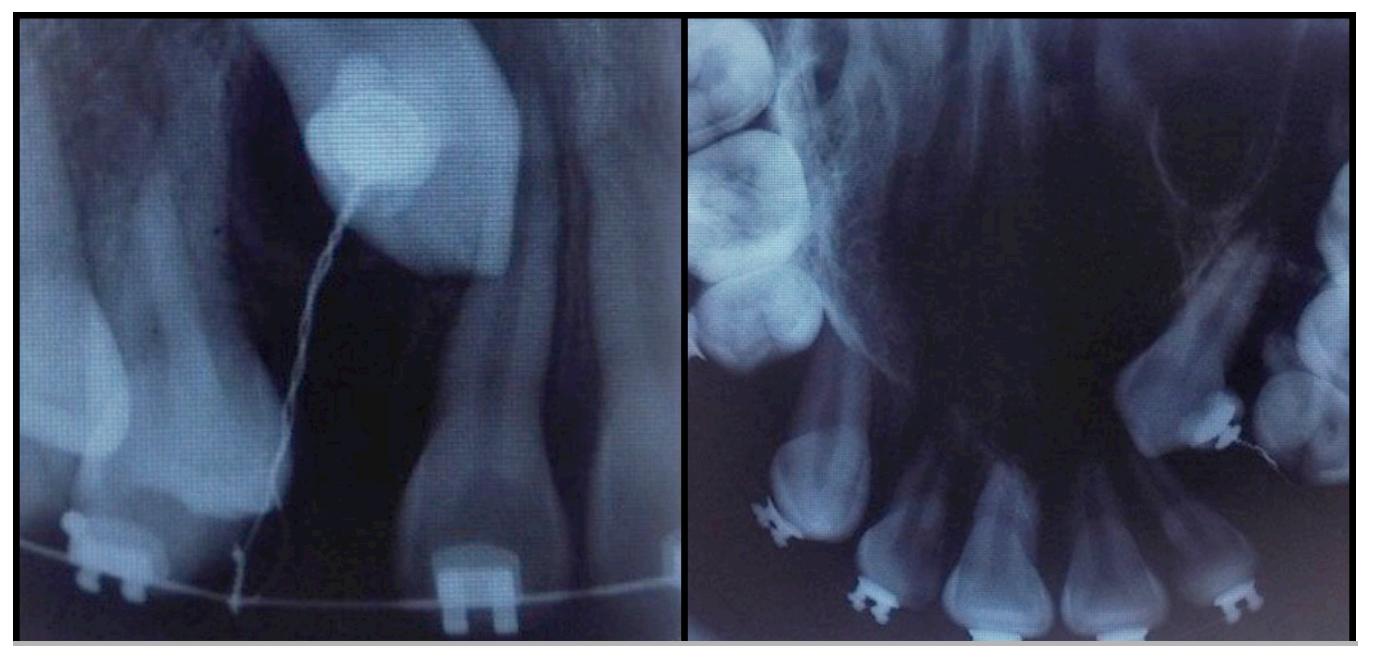

Figura 5. Control radiográfico postquirúrgico a los 10 días 


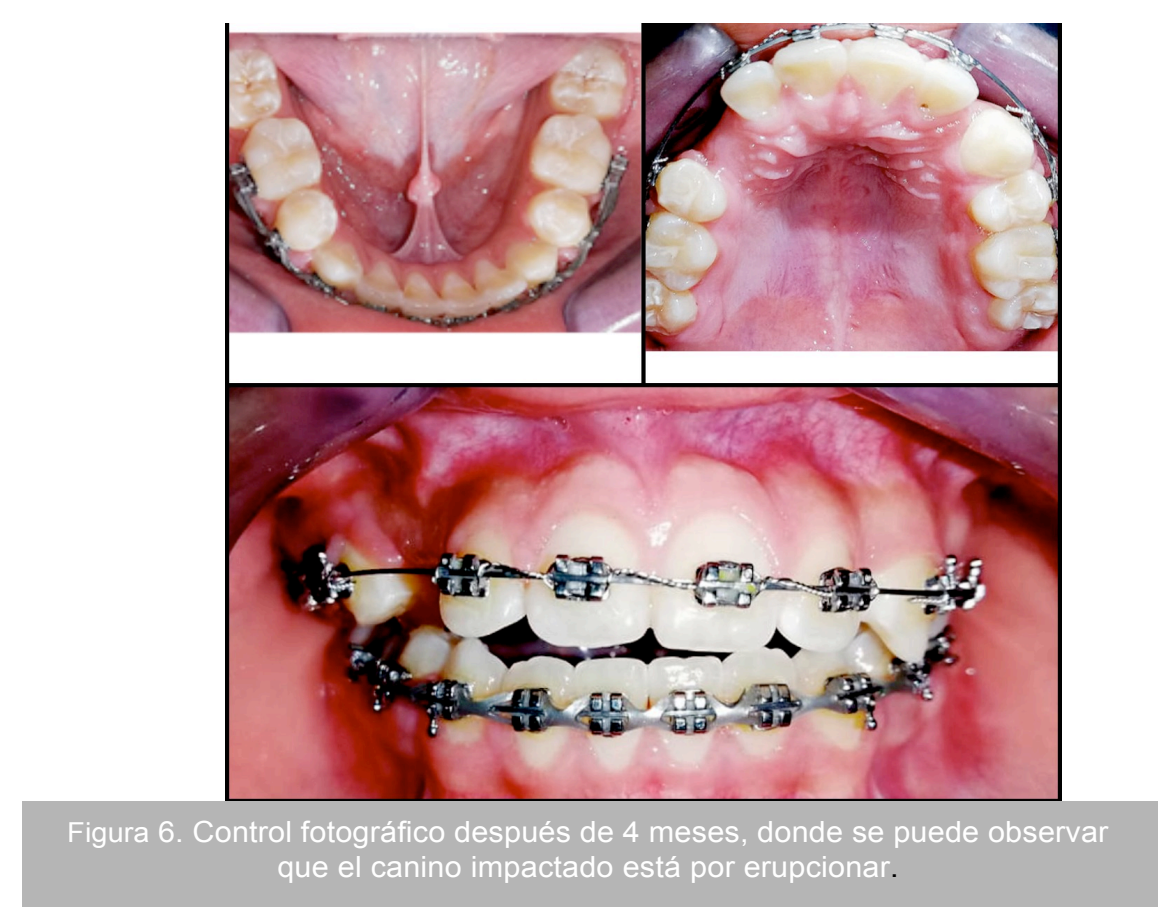

Después de 4 meses se observó un descenso importante del canino maxilar impactado, y se realizó una incisión gingival vertical, para facilitar su erupción. Tras comprobar que había una adecuada evolución del canino, se realizó la exodoncia de la pieza \#1.4 para proporcionar el espacio necesario.

\section{DISCUSIÓN}

Harris et $a^{(3)}$, en su estudio publicaron que estas lesiones se descubren con el examen radiográfico de rutina, el motivo de consulta más habitual es la falta de erupción de un diente permanente, con o sin la persistencia del diente temporal, Palacios ${ }^{(1)}$ en su estudio publica que estas lesiones se diagnostican de forma casual mediante exámenes radiográficos de rutina entre la segunda y tercera década de la vida o bien a partir de algún signo clínico como retraso de la erupción dentaria, Flores et al ${ }^{(9)}$ publicaron que los odontomas suelen ser asintomáticos y su hallazgo suele ser casual tras realizar un estudio radiográfico habitual, generalmente entre la primera y la segunda década de la vida; coincidiendo con este caso, cuyo motivo de consulta fue la falta de erupción de la pieza \# 13, permanencia del diente deciduo \# 53 y que fue un hallazgo radiográfico.

Blanco et al ${ }^{(5)}$ en su estudio publicó que pueden aparecer signos y síntomas relacionados con su presencia como son dientes supernumerarios, incisivos impactados, caninos impactados, molares impactados, inflamación e infección, obstrucción nasal, coincidiendo con el caso la inclusión del canino.

Con relación a la localización de los odontomas Palacios et al ${ }^{(1)}$, en su estudio reporta que hay una predilección en el maxilar del $67,4 \%$ y en la mandíbula del $32.6 \%$, Avelar et al ${ }^{(7)}$, en su estudio reporta que hay una predilección de $57,5 \%$ en maxilar y $42,5 \%$ en mandíbula, Puello et al ${ }^{(8)}$ y Harris ${ }^{(2)}$ reportan en su estudio que los odontomas compuestos principalmente se localizan en la región anterior del maxilar superior y los odontomas complejos principalmente se localizan en la región posterior de la mandíbula, coincidiendo con este caso la localización del odontoma compuesto en la región anterior maxilar.

Flores et al ${ }^{(9)}$ y Thistle et al ${ }^{(11)}$, sugieren un tratamiento quirúrgico-ortodóncico: quirúrgico para el odontoma y ortodóncico para el canino incluido el cual se reposicionará en la arcada, coincidiendo con el caso la elección del tratamiento integral quirúrgico-ortodóncico.

Harris et al ${ }^{(2)}$, Harris et al ${ }^{(3)}$, Moya ${ }^{(6)}$ y Blanco et al (5), sugieren un tratamiento netamente quirúrgico incluyendo enucleación y eliminando la cápsula de tejido conjuntivo que rodea 
al odontoma, estos tratamientos según los estudios se realizaron en personas que cursaban la tercera década de la vida y no tenían piezas dentarias incluidas. En el caso presentado se encontró además del odontoma una pieza dentaria incluida, es por eso que se descartó este tratamiento.

Blanco et al ${ }^{(5)}$, reporta como tratamiento la exodoncia del odontoma y de canino retenido por no ser viable para su posicionamiento en la arcada, Loughney et al ${ }^{(12)}$ en su estudio reporta como tratamiento exodoncia del odontoma y de pieza dentaria retenida por que la inclusión dentaria es alta y la raíz presenta rizólisis, en el caso clínico presentado el canino incluido no presentaba estas características y era viable para su posicionamiento en la arcada, por lo cual se descartó el tratamiento de exodoncia de la pieza incluida.

Los odontomas son tumores benignos generalmente asintomáticos, la razón de consulta más frecuente es el retraso de la erupción dental permanente asociada o no, a la persistencia del diente temporal, el estudio radiográfico es de suma importancia para su diagnóstico. El tratamiento acertado siempre será la extirpación del odontoma, y si hay presencia de piezas dentarias incluidas, y si su pronóstico es favorable y viable, se procederá a su conservación.

Contribución de autoría: Los autores MUL y ACG, desarrollaron el caso, redactaron, y aprobaron la versión final del manuscrito.

Fuente de financiamiento: El trabajo fue financiado por los autores.

Conflicto de intereses: Los autores declararon no tener conflictos de interés en la publicación del presente artículo.

\section{REFERENCIAS BIBLIOGRAFICAS}

1. Palacios, D., Guzmán, B., Miranda, J., \& Ramos, C. Odontoma compuesto: revisión de la literatura y reporte de un caso con 40 dentículos. Revista ADM. 2016;73(4):206-211.
2. Harris, R., Diaz, A., \& Carbonell, Z. Odontoma compuesto. Una patologia oral a considerar. Duazary. 2010; 7(1) 91-94.

3. Harris, R., Rebolledo, M., Diaz, A., \& Carbonell, Z. Odontoma serie de casos. Revisión de literatura. Avances en Odontoestomatologia. 2011; 27(11): 25-32.

4. Barnes, L., Eveson, J., Reichart, P., \& Sidransky, D. (2005). Pathology and Genetics of Head and Neck Tumours . Lyon.

5. Blanco, G., Gneco, B., Lozano, F., Pérez, L., \& Chaverra, M. Odontoma compuesto maxilar, reporte de un caso y revisión de la literatura. Acta Odontológica Venezolana. 2011; 49(2)

6. Moya, Z. Diagnóstico precoz y tratamento oportuno del odontoma compuesto en un niño Peruano de 3 años. Odontología Pediátrica. 2016; 15(2):155-161

7. Avelar, R., Azoubel, A., Santos, T., Souza, E., \& Dourado, E. Odontogenic tumors: clinical and pathology study of 238 cases. Revista brasileña Otorrinolaringologia. 2008; 74(5): 668-673.

8. Puello, E., Sir, F., \& Carbal, A. Odontomas: reporte y serie de casos clinicos. Revista Odontologica Mexicana. 2017; 21(3) 214-217.

9. Flores, R., Simón, E., Torres, D., Yáñez, R., \& Gutiérrez, J. Odontoma compuesto asociado a canino permanente inferior incluido. Gaceta Dental. 2013; 244:120-127.

10. Escoda, C. G., \& Aytés, L. B. Tratado de cirugia bucal. 1th. Ed. España: Ergon; 2003.

11. Thistle, L., Muela, D., Nevárez, M., Rios, V., \& Nevárez, A. Aspectos descriptivos del odontoma: revisión de la literatura. Elsevier [internet] 2016 [consultado 3 de Ene 2018] 20(4). Disponible en: https://www.elsevier.es/es-revista-revistaodontologica-mexicana-90-articulo-aspectosdescriptivos-del-odontoma-revisionS1870199X16300611

12. Loughney, A., Loughney, JC., Vila, B., Fernández, M. Odontoma compuesto. Cient Dent [Internet] 2011 [consultado 4 Ene 2018] 8;2:123-126. Disponible en: https://www.coem.org.es/sites/default/files/pub licaciones/CIENTIFICA_DENTAL/vol8_num2/ 43-46.pdf

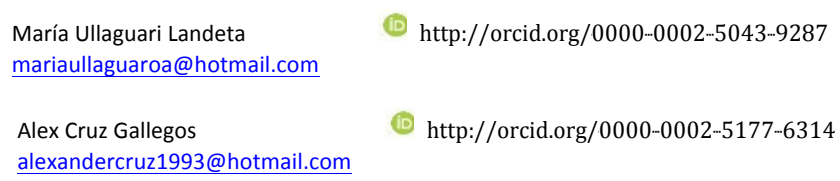

Copyright $($ La revista. La revista Kiru es publicada por la Facultad de Odontología de la Universidad de San Martín de Porres, en Lima, Perú. 